\title{
On $(2-d)$-kernels in the cartesian product of graphs
}

\begin{abstract}
In this paper we study the problem of the existence of $(2-d)$ kernels in the cartesian product of graphs. We give sufficient conditions for the existence of $(2-d)$-kernels in the cartesian product and also we consider the number of $(2-d)$-kernels.
\end{abstract}

1. Introduction. In general we use the standard terminology and notation of graph theory, see [3]. Graphs $G=(V(G), E(G))$ considered in this paper are undirected, connected and simple. By $P_{n}, n \geq 2$ and $C_{n}, n \geq 3$ we mean a path and a cycle on $n$ vertices, respectively. By $d_{G}(x, y)$ we denote the distance between vertices $x$ and $y$ in $G$ being the length of the shortest path from $x$ to $y$. Consequently $d_{G}(X, Y)=\min \left\{d_{G}(u, v): u \in X, v \in Y\right\}$ means the distance between sets $X$ and $Y$. A simple graph $G=G\left(V_{1}, V_{2}\right)$ is called bipartite if its vertex set can be partitioned into two disjoint subsets $V_{1}, V_{2}$ such that every edge has the form $x y$, where $x \in V_{1}$ and $y \in V_{2}$.

Let $G_{1}=\left(V_{1}, E_{1}\right)$ and $G_{2}=\left(V_{2}, E_{2}\right)$ be two graphs. The cartesian product of two graphs $G_{1}$ and $G_{2}$ is the graph $G_{1} \times G_{2}$ such that $V\left(G_{1} \times\right.$ $\left.G_{2}\right)=V_{1} \times V_{2}$ and $E\left(G_{1} \times G_{2}\right)=\left\{\left(x_{i}, y_{p}\right)\left(x_{j}, y_{q}\right):\left(x_{i}=x_{j}\right.\right.$ and $y_{p} y_{q} \in$ $\left.E\left(G_{2}\right)\right)$ or $\left(y_{p}=y_{q}\right.$ and $\left.\left.x_{i} x_{j} \in E\left(G_{1}\right)\right)\right\}$.

By the cartesian product of $n$ graphs $G_{1}, \ldots, G_{n}$ we mean the cartesian product of $G_{n}$ and $G_{1} \times G_{2} \times \ldots \times G_{n-1}$ denoted by $G_{1} \times G_{2} \times \ldots \times G_{n}$. If $n=2$, then we obtain the definition of cartesian product of two graphs.

2010 Mathematics Subject Classification. 05C20, 05C69.

Key words and phrases. Independence, domination, cartesian product, $(2-d)$-kernel. 
Theorem 1.1 ([12]). A graph $G_{1} \times G_{2}$ is bipartite if and only if $G_{1}$ and $G_{2}$ are bipartite.

We say that a subset $D \subseteq V(G)$ is dominating, if every vertex of $G$ is either in $D$ or it is adjacent to at least one vertex of $D$. A subset $S \subseteq V(G)$ is independent if no two vertices of $S$ are adjacent in $G$. A subset $J$ being dominating and independent is a kernel of $G$.

H. Galeana-Sánchez and C. Hernández-Cruz played an important role in researching kernels in digraphs. During the last decades they studied not only kernels in digraphs but some of its generalizations, mainly kernels by monochromatic paths and $(k, l)$-kernels. Most of the existing results about kernels and their generalizations in digraphs were related to operations in digraphs and how the kernels are preserved. For results concerning kernels which were obtained quite recently see $[4,6,7,5,8,9,10,11]$.

In [17] A. Włoch introduced and studied the concept of a 2-dominating kernel (for convenience we will write shortly $(2-d)$-kernel). A set $J$ is a $(2-d)$-kernel of a graph $G$ if it is independent and 2-dominating, i.e. $J$ is independent and each vertex from $V(G) \backslash J$ has at least two neighbours in $J$.

Not every graph possesses a $(2-d)$-kernel, for example a graph $P_{4}$ is a graph without $(2-d)$-kernel. In [1] it was proved that the problem of the existence of $(2-d)$-kernel is $\mathcal{N} \mathcal{P}$-complete for a general graph.

Some results related to the existence of $(2-d)$-kernels in graphs can be found in [1], [2] and [17]. Moreover, in [1] the number of all $(2-d)$-kernels (denoted by $\sigma_{(2-d)}(G)$ ) in graphs was studied. In this paper we consider the problem of the existence of $(2-d)$-kernels in the cartesian product of graphs and also we consider the number of $(2-d)$-kernels in this graph product.

The topic of kernels in graphs product was studied for example in [14, $13,15,16,17,18,19]$.

2. Main results. In this section we give some necessary and sufficient conditions for the existence of $(2-d)$-kernels in the cartesian product of graphs.

Theorem 2.1. If $G$ and $H$ are connected bipartite graphs, then $G \times H$ has two disjoint $(2-d)$-kernels.

Proof. Let $G=G\left(V_{1}, V_{2}\right)$ and $H=H\left(V_{3}, V_{4}\right)$ be bipartite. Then the set $V(G \times H)$ is the union of pairwise disjoint sets $V_{1} \times V_{3}, V_{1} \times V_{4}, V_{2} \times V_{3}$ and $V_{2} \times V_{4}$. We shall show that sets $J=\left(V_{1} \times V_{3}\right) \cup\left(V_{2} \times V_{4}\right)$ and $J^{*}=\left(V_{2} \times V_{3}\right) \cup\left(V_{1} \times V_{4}\right)$ are $(2-d)$-kernels of the graph $G \times H$. Firstly we shall show that $J$ is a $(2-d)$-kernel. To prove that $J$ is independent let us assume that $\left(x_{i}, y_{j}\right),\left(x_{p}, y_{q}\right) \in J$ and consider the following cases:

$1.1\left(x_{i}, y_{j}\right),\left(x_{p}, y_{q}\right) \in V_{1} \times V_{3}$. If $i=p$, then by the definition of $H$ and the cartesian product 
$G \times H$ we have that $y_{j}, y_{q} \in V_{3}$, so $y_{j} y_{q} \notin E(H)$ and consequently $\left(x_{i}, y_{j}\right)\left(x_{p}, y_{q}\right) \notin E(G \times H)$.

If $j=q$, then we prove analogously with respect to the graph $G$.

Let $i \neq p$ and $j \neq q$. Then the definition of $G \times H$ immediately gives that $\left(x_{i}, y_{j}\right)\left(x_{p}, y_{q}\right) \notin E(G \times H)$.

$1.2\left(x_{i}, y_{j}\right),\left(x_{p}, y_{q}\right) \in V_{2} \times V_{4}$.

Then we prove in the same way as in 1.1.

$1.3\left(x_{i}, y_{j}\right) \in V_{1} \times V_{3}$ and $\left(x_{p}, y_{q}\right) \in V_{2} \times V_{4}$.

Since $G$ and $H$ are bipartite, so $x_{i} x_{p} \notin E(G)$ and $y_{j} y_{q} \notin E(H)$. Then by the definition of $G \times H$ we have that $\left(x_{i}, y_{j}\right)\left(x_{p}, y_{q}\right) \notin$ $E(G \times H)$.

Consequently $J$ is independent. Now we shall prove that $J$ is 2-dominating. Assume that $\left(x_{i}, y_{j}\right) \notin J$. It suffices to show that there are two vertices from the set $J$ which dominate the vertex $\left(x_{i}, y_{j}\right)$. We consider the following possibilities:

$2.1\left(x_{i}, y_{j}\right) \in V_{1} \times V_{4}$.

Then $x_{i} \in V_{1}$ and $y_{j} \in V_{4}$. Because $H$ is connected and bipartite so there is a vertex, say $y_{q} \in V_{3}$ such that $y_{j} y_{q} \in E(H)$. Consequently by the definition of $G \times H$ we have that there is $\left(x_{i}, y_{q}\right) \in V_{1} \times V_{3} \subset$ $V(G \times H)$. This means that $\left(x_{i}, y_{q}\right) \in J$ and $\left(x_{i}, y_{j}\right)\left(x_{i}, y_{q}\right) \in E(G \times$ $H)$. Analogously with respect to graph $G$ we can show that there exists a vertex $\left(x_{p}, y_{j}\right) \in V_{2} \times V_{3} \subset V(G \times H)$. So $\left(x_{p}, y_{j}\right) \in J$ and $\left(x_{i}, y_{j}\right)\left(x_{p}, y_{j}\right) \in E(G \times H)$. Thus every vertex from the set $V_{1} \times V_{4}$ is at least 2-dominated by the set $J$.

$2.2\left(x_{i}, y_{j}\right) \in V_{2} \times V_{3}$,

Then we prove by using the same method as in 2.1.

Finally, the set $J$ is a $(2-d)$-kernel of $G \times H$. In the same way we can prove that $J^{*}$ is a $(2-d)$-kernel of $G \times H$ and it is obvious that $J \cap J^{*}=\emptyset$, which ends the proof.

Using Theorems 1.1 and 2.1, we can prove the result for the cartesian product of $n$ graphs.

Theorem 2.2. Let $n \geq 2$ be an integer. If $G_{i}$ is bipartite, for $i=1, \ldots, n$, then $G_{1} \times G_{2} \times \ldots \times G_{n}$ has two disjoint $(2-d)$-kernels.

From the above follow results for special bipartite graphs.

Corollary 2.3. Let $n, m \geq 2$ be integers. A graph $P_{n} \times P_{m}$ has exactly two disjoint $(2-d)$-kernels $J_{1}, J_{2}$ and $J_{1} \cup J_{2}=V\left(P_{n} \times P_{m}\right)$. Moreover, $\left|J_{1}\right|=\left|J_{2}\right|=\frac{m \cdot n}{2}$ if $m \cdot n$ is even and $\left|J_{1}\right|=\left\lfloor\frac{m \cdot n}{2}\right\rfloor$ and $\left|J_{2}\right|=\left\lceil\frac{m \cdot n}{2}\right\rceil$, otherwise.

Corollary 2.4. Let $n, m \geq 2$ be integers. A graph $C_{2 n} \times P_{m}$ has exactly two disjoint $(2-d)$-kernels $J_{1}, J_{2}$ and $J_{1} \cup J_{2}=V\left(C_{2 n} \times P_{m}\right)$. Moreover, $\left|J_{1}\right|=\left|J_{2}\right|=m \cdot n$. 
Theorem 2.5. Let $n, m \geq 3$ be integers. A graph $C_{n} \times C_{m}$ has a $(2-d)$ kernel if and only if $n$ and $m$ are even or $n=m$.

Proof. Let $n, m$ be as in the statement of the theorem. If $n$ and $m$ are even, then $C_{n}$ and $C_{m}$ are bipartite and $C_{n} \times C_{m}$ has a $(2-d)$-kernel by Theorem 2.1. Assume that $n=m$ and $n$ is odd. We will prove that $C_{n} \times C_{n}$ has a $(2-d)$-kernel $J$. Let $V\left(C_{n}\right)=\left\{x_{1}, \ldots, x_{n}\right\}, n \geq 3$ with the numbering of vertices in the natural fashion. We can illustrate the construction of the set $J$ in $C_{n} \times C_{n}$ using the matrix $A=\left[a_{i j}\right]_{n \times n}$ defined as follows

$$
a_{i j}=\left\{\begin{array}{l}
1 \text { if the vertex }\left(x_{i}, x_{j}\right) \in J \\
0 \text { otherwise }
\end{array}\right.
$$

Then $a_{i j}=1$ if and only if

$$
i-j \in\left\{-n+2 p ; p=1, \ldots, \frac{n-3}{2}\right\} \cup\left\{n-2 q-1 ; q=1, \ldots, \frac{n-1}{2}\right\}
$$

and $a_{i j}=0$ otherwise. For the explanation if $n=3$, then

$$
A=\left[\begin{array}{lll}
1 & 0 & 0 \\
0 & 1 & 0 \\
0 & 0 & 1
\end{array}\right] .
$$

Let $n \geq 5$ be odd. Then the matrix $A$ has the form

$$
A=\left[\begin{array}{cccccccccc}
1 & 0 & 0 & 1 & 0 & \ldots & 1 & 0 & 1 & 0 \\
0 & 1 & 0 & 0 & 1 & \ldots & 0 & 1 & 0 & 1 \\
1 & 0 & 1 & 0 & 0 & \ldots & 1 & 0 & 1 & 0 \\
0 & 1 & 0 & 1 & 0 & \ldots & 0 & 1 & 0 & 1 \\
1 & 0 & 1 & 0 & 1 & \ldots & 1 & 0 & 1 & 0 \\
\vdots & \vdots & \vdots & \vdots & \vdots & \ddots & \vdots & \vdots & \vdots & \vdots \\
0 & 1 & 0 & 1 & 0 & \ldots & 1 & 0 & 0 & 1 \\
1 & 0 & 1 & 0 & 1 & \ldots & 0 & 1 & 0 & 0 \\
0 & 1 & 0 & 1 & 0 & \ldots & 1 & 0 & 1 & 0 \\
0 & 0 & 1 & 0 & 1 & \ldots & 0 & 1 & 0 & 1
\end{array}\right]
$$

Conversely suppose on the contrary that $C_{n} \times C_{m}$ has a $(2-d)$-kernel, say $J$ where $m \neq n$ and $n$ is odd.

Let $m$ be even and $n$ be odd. Then the graph $C_{m}$ has two disjoint $(2-d)$ kernels $J_{1}$ and $J_{2}$. Let $J_{i}^{(p)}, i=1,2$ be a $(2-d)$-kernel in $C_{m}^{(p)}$, where $C_{m}^{(p)} \subset C_{m} \times C_{n}$ is a $p$-copy of $C_{m}, p=1, \ldots, m$. It is clear that $J=J_{1}^{(1)} \cup$ $J_{2}^{(2)} \cup J_{1}^{(3)} \cup \ldots \cup J_{1}^{(n)}$, because $n$ is odd, but then $d_{C_{m} \times C_{n}}\left(J_{1}^{(1)}, J_{1}^{(n)}\right)=1$, a contradiction with the independence of $J$.

Let now $m \neq n$ and $m, n$ be odd. Since $m \neq n$, so without loss of the generality let $m>n$. Suppose that $C_{m} \times C_{n}$ has a $(2-d)$-kernel $J$. By 
definition of $C_{m} \times C_{n}$ and $(2-d)$-kernel it is obvious that in each copy $C_{m}^{(i)}$, $i=1, \ldots, n$ of $C_{m} \times C_{n}$ we have to choose a maximal independent set $J_{i}$ such that $\left|J_{i}\right|=\left\lfloor\frac{m}{2}\right\rfloor$, for all $i=1, \ldots, n$ and $J_{i}$ is a subset of $(2-d)$-kernel $J$. Using given earlier construction of $(2-d)$-kernel $J$ in $C_{m} \times C_{n}$ which preserve 2-domination we observe that in copy $C_{m}^{(n)}$ for every maximal independent set $J_{n}$ the set $\bigcup_{i=1}^{n} J_{i}$ is not independent, which is a contradiction with the assumption.

Corollary 2.6. Let $m, n \geq 3$ be integers. If $m, n$ are even, then $\sigma_{(2-d)}\left(C_{n} \times\right.$ $\left.C_{m}\right)=2$. If $n$ is odd, then $\sigma_{(2-d)}\left(C_{n} \times C_{n}\right)=2 n$.

Corollary 2.6 follows by the proof of Theorem 2.5.

Theorem 2.7. Let $m, n \geq 1$ be integers. A graph $K_{n} \times K_{m}$ has a $(2-d)$ kernel if and only if $n=m$.

Proof. Let $V\left(K_{n}\right)=\left\{x_{1}, \ldots, x_{n}\right\}, n \geq 1$ and $V\left(K_{m}\right)=\left\{y_{1}, \ldots, y_{m}\right\}, m \geq$ 1. If $m=n$, then it is easy to observe that the set $J=\left\{\left(x_{i}, y_{i}\right), i=1, \ldots, n\right\}$ is $(2-d)$-kernel of the graph $K_{n} \times K_{n}$.

Suppose on the contrary that a graph $K_{n} \times K_{m}$ has a $(2-d)$-kernel $J^{*}$ and $n>m$. Clearly in each copy of a complete graph $K_{n}$ and $K_{m}$ we can choose at most one vertex to the set $J^{*}$. Without loss of the generality let $\left(x_{1}, y_{1}\right) \in J^{*}$. Then in the copy $K_{m}^{(2)}$ we choose an arbitrary vertex $\left(x_{2}, y_{i}\right)$ where $i \neq 1$ and $\left(x_{2}, y_{i}\right) \in J^{*}$. Analogously in the copy $K_{m}^{(3)}$ we choose a vertex $\left(x_{3}, y_{j}\right) \in J^{*}$ for $j \neq 1$ and $j \neq i$. Consequently in the copy $K_{n+1}^{(i)}$ for every vertex $\left(x_{n+1}, y_{p}\right), p=1, \ldots, m$ there is a vertex $\left(x_{r}, y_{p}\right)$ where $1 \leq r \leq m$. Then $J^{*} \cup\left\{\left(x_{n+1}, y_{p}\right), 1 \leq p \leq n\right\}$ is not independent, which is a contradiction with the assumption that $J^{*}$ is a $(2-d)$-kernel.

Thus the theorem is proved.

Corollary 2.8. Let $n \geq 2$ be integer. Then $\sigma_{(2-d)}\left(K_{n} \times K_{n}\right)=n$ !. Moreover, all $(2-d)$-kernels of $K_{n} \times K_{n}$ have the same cardinality $n$.

Proof. Let $J \subset V\left(K_{n} \times K_{n}\right)$ be a $(2-d)$-kernel. Then it is clear that in the copy $K_{n}^{(1)}$ we can choose the vertex belonging to $J$ on $n$ ways. Moreover, in the copy $K_{n}^{(p)}, 2 \leq p \leq n$ we can choose the vertex belonging to $J$ on $(n-p+1)$ ways. This gives that $\sigma_{(2-d)}\left(K_{n} \times K_{n}\right)=n(n-1) \cdot \ldots \cdot 1=n$ !.

Let $X \subset V(G)$. A graph $G$ is $X-(2-d)$-kernel critical if $G \backslash X$ has a $(2-d)$-kernel.

Let $X, Y \subset V(G)$ be two disjoint subsets of $G$. A graph $G$ is $(X, Y)$ $(2-d)$-kernel critical if $Y$ is a $(2-d)$-kernel of $G \backslash X$.

If a graph $G$ is $(X, Y)-(2-d)$-kernel critical and $(Y, X)-(2-d)$-kernel critical, then we will write that $G$ is an $\{X, Y\}-(2-d)$-kernel critical. 
Theorem 2.9. Let $G=G\left(J_{1}, J_{2}\right)$ be a bipartite graph such that $J_{i}, i=1,2$ are $(2-d)$-kernels of $G$. Let $H$ be a graph with $V_{1}, V_{2} \subset V(H)$ such that $H$ is $\left\{V_{1}, V_{2}\right\}-(2-d)$-kernel critical. Then $G \times H$ has at least two $(2-d)$-kernels.

Proof. Let $G=G\left(J_{1}, J_{2}\right)$ and $H$ be as in the statement of the theorem. If $V_{1} \cup V_{2}=V(H)$, then graphs $G$ and $H$ are bipartite and by Theorem 2.1, a graph $G \times H$ has two $(2-d)$-kernels. Suppose that $V_{1} \cup V_{2} \neq V(H)$. Let $R=V(H) \backslash\left(V_{1} \cup V_{2}\right)$. Then the set $V(G \times H)$ is the union of pairwise disjoint sets $J_{1} \times V_{1}, J_{1} \times V_{2}, J_{1} \times R, J_{2} \times V_{1}, J_{2} \times V_{2}$ and $J_{2} \times R$. We will prove that set $J=\left(J_{1} \times V_{1}\right) \cup\left(J_{2} \times V_{2}\right)$ is $(2-d)$-kernel of the graph $G \times H$. Firstly we show that $J$ is an independent set.

Let $\left(x_{i}, y_{j}\right),\left(x_{p}, y_{q}\right) \in J$ and consider the following cases:

$1.1\left(x_{i}, y_{j}\right),\left(x_{p}, y_{q}\right) \in J_{1} \times V_{1}$.

If $j=q$, then by the definition of $G$ and the cartesian product $G \times H$ we have that $x_{i}, x_{p} \in J_{1}$, so $x_{i}, x_{p} \notin E(G)$ and consequently $\left(x_{i}, y_{j}\right)\left(x_{p}, y_{q}\right) \notin E(G \times H)$.

Let $i=p$. Since a graph $H$ is $\left\{V_{1}, V_{2}\right\}$ - $(2-d)$-kernel critical, then $V_{1}$ is $(2-d)$-kernel of $H \backslash V_{2}$ and this means that $V_{1}$ is an independent set of a graph $H$. So for all $y_{j}, y_{q} \in V_{1}$ we have that $y_{j} y_{q} \notin E(H)$ and finally $\left(x_{i}, y_{j}\right)\left(x_{p}, y_{q}\right) \notin E(G \times H)$.

Let $i \neq p$ and $j \neq q$. Then the definition of $G \times H$ immediately gives that $\left(x_{i}, y_{j}\right)\left(x_{p}, y_{q}\right) \notin E(G \times H)$.

$1.2\left(x_{i}, y_{j}\right),\left(x_{p}, y_{q}\right) \in J_{2} \times V_{2}$.

Then we prove in the same way as in 1.1.

$1.3\left(x_{i}, y_{j}\right) \in J_{1} \times V_{1}$ and $\left(x_{p}, y_{q}\right) \in J_{2} \times V_{2}$.

Since $G$ is bipartite and $H$ is $\left\{V_{1}, V_{2}\right\}-(2-d)$-kernel critical so $x_{i} x_{p} \notin$ $E(G)$ and $y_{j} y_{q} \notin E(H)$. Then by the definition of $G \times H$ we have that $\left(x_{i}, y_{j}\right)\left(x_{p}, y_{q}\right) \notin E(G \times H)$.

Consequently $J$ is independent. Let $\left(x_{i}, y_{j}\right) \notin J$. We prove that $\mathrm{J}$ is 2 dominating. Consider the following cases:

2.1. $\left(x_{i}, y_{j}\right) \in J_{2} \times V_{1}$,

Then $x_{i} \in J_{2}$ and $y_{j} \in V_{1}$. Because $J_{1}$ is $(2-d)$-kernel of a graph $G$ then there exist at least two vertices, say $x_{s}, x_{r}$ such that $x_{s} x_{i}, x_{r} x_{i} \in$ $E(G)$. By the definition of cartesian product we have that every vertex from $J_{2} \times V_{1}$ is at least 2 -dominated by the set $J_{1} \times V_{1}$.

2.2. $\left(x_{i}, y_{j}\right) \in J_{1} \times V_{2}$,

We can prove this using the same method as in 2.1.

2.3. $\left(x_{i}, y_{j}\right) \in J_{1} \times R$ or $\left(x_{i}, y_{j}\right) \in J_{2} \times R$,

Then $x_{i} \in V(G)$ and $y_{j} \in R$. Because the graph $H$ is $\left\{V_{1}, V_{2}\right\}$ $(2-d)$-kernel critical then $V_{1}$ is $(2-d)$-kernel of $H \backslash V_{2}$ and there exist at least two vertices, say $y_{w}, y_{t}$ such that $y_{w} y_{j}, y_{t} y_{j} \in E(H)$. Moreover every vertex from the set $V_{1} \times R$ is at least 2-dominated by 
the set $J_{1} \times V_{1}$. Analogously, considering set $V_{2}$, we can show that every vertex from $J_{2} \times R$ is at least 2 -dominated by the set $J_{2} \times V_{2}$. All this together gives that $J$ is a $(2-d)$-kernel of $G \times H$. In the same way we can prove that $J^{*}=J_{1} \times V_{2} \cup J_{2} \times V_{1}$ is a $(2-d)$-kernel of $G \times H$, which ends the proof.

3. Conclusion and further study. The problem of finding the characterization of the cartesian product $G \times H$ with a $(2-d)$-kernel is still open. However, some sufficient conditions can be found if we add a restriction that a graph $G$ is fixed.

\section{REFERENCES}

[1] Bednarz, P., Hernández-Cruz, C., Włoch, I., On the existence and the number of $(2-d)$-kernels in graphs, Ars Combin. 121 (2015), 341-351.

[2] Bednarz, P., Włoch, I., An algorithm determining $(2-d)$-kernels in trees, Util. Math., in print.

[3] Diestel, R., Graph Theory, Springer-Verlag, Heidelberg, New York, 2005.

[4] Galeana-Sánchez, H., Gómez, R., $(k, l)$-kernels, $(k, l)$-semikernels, $k$-Grundy functions and duality for state splittings, Discuss. Math. Graph Theory 27 (2007), 359371.

[5] Galeana-Sánchez, H., Hernández-Cruz, C., On the existence of k-kernels in digraphs and in weighted digraphs, AKCE Int. J. Graphs Comb. 7 (2) (2010), 201-215.

[6] Galeana-Sánchez, H., Hernández-Cruz, C., k-kernels in generalizations of transitive digraphs, Discuss. Math. Graph Theory 31 (2) (2011), 293-312.

[7] Galeana-Sánchez, H., Hernández-Cruz, C., Cyclically k-partite digraphs and $k$ kernels, Discuss. Math. Graph Theory 31 (1) (2011), 63-78.

[8] Galeana-Sánchez, H., Hernández-Cruz, C., On the existence of $(k, l)$-kernels in infinite digraphs: A survey, Discuss. Math. Graph Theory 34 (3) (2014), 431-466.

[9] Galeana-Sánchez, H., Pastrana-Ramírez, L., Extending digraphs to digraphs with (without) k-kernel, Int. J. Contemp. Math. Sci. 3 (5) (2008), 229-243.

[10] Galeana-Sánchez, H., Pastrana-Ramírez, L., k-kernels in the orientation of the path graph, Int. J. Contemp. Math. Sci. 5 (5) (2010), 231-242.

[11] Galeana-Sánchez, H., Pastrana-Ramírez, L., A construction that preserves the number of k-kernels, Int. J. Contemp. Math. Sci. 6 (10) (2011), 491-502.

[12] Imrich, W., Klavžar, S., Rall, D. F., Topics in Graph Theory: Graphs and Their Cartesian Product, A. K. Peters Ltd., Wellesley Massachusetts, 2008.

[13] Kucharska, M., Kwaśnik, M., On $(k, l)$-kernels of special superdigraphs of $P_{m}$ and $C_{m}$, Discuss. Math. Graph Theory 21 (1) (2001), 95-109.

[14] Kwaśnik, M., $(k, l)$-kernels in graphs and in their products, Ph.D. Dissertation, Wrocław, 1980.

[15] Szumny, W., Włoch, A., Włoch, I., On $(k, l)$-kernels in D-join of digraphs, Discuss. Math. Graph Theory 27 (2007), 457-470.

[16] Szumny, W., Włoch, A., Włoch, I., On the existence and on the number of $(k, l)$ kernels in the lexicographic product of graphs, Discrete Math. 308 (20) (2008), 46164624.

[17] Włoch, A., On 2-dominating kernels in graphs, Australas. J. Combin. 53 (2012), $273-284$.

[18] Włoch, A., Włoch, I., On $(k, l)$-kernels in generalized products, Discrete Math. 164 (1997), 295-301. 
[19] Włoch, A., Włoch, I., On $(k, l)$-kernels in the corona of digraphs, Int. J. Pure Appl. Math. 53 (4) (2009), 571-582.

Paweł Bednarz

The Faculty of Mathematics and Applied Physics

Rzeszów University of Technology al. Powstańców Warszawy 8 35-959 Rzeszów

Poland

e-mail: pbednarz@prz.edu.pl

Received August 9, 2016
Iwona Włoch

The Faculty of Mathematics and Applied Physics

Rzeszów University of Technology

al. Powstańców Warszawy 8

35-959 Rzeszów

Poland

iwloch@prz.edu.pl 